\title{
Current fluctuations in the weakly asymmetric exclusion process with open boundaries
}

\author{
Mieke Gorissen ${ }^{1}$ and Carlo Vanderzande ${ }^{1,2}$ \\ ${ }^{1}$ Faculty of Sciences, Hasselt University, 3590 Diepenbeek, Belgium \\ ${ }^{2}$ Instituut Theoretische Fysica, KULeuven, 3001 Heverlee, Belgium
}

(Received 3 February 2012; revised manuscript received 10 October 2012; published 15 November 2012)

\begin{abstract}
The additivity principle allows a calculation of current fluctuations and associated density profiles in large diffusive systems. We apply this principle to the weakly asymmetric exclusion process (WASEP). We also calculate the cumulant generating function of the current and the density profiles associated with rare currents in finite systems using a numerical approach based on the density matrix renormalization group. Comparison of the two approaches allows us to verify the validity of the additivity principle and to get insight into the finite size scaling theory for current fluctuations in the WASEP. No evidence for a dynamical phase transition is found.
\end{abstract}

DOI: 10.1103/PhysRevE.86.051114

PACS number(s): 05.40.-a, 02.50.-r, 05.70.Ln

\section{INTRODUCTION}

Systems driven out of equilibrium by putting them in contact with reservoirs at different chemical potential or temperature develop currents. It is a main problem of nonequilibrium statistical mechanics to determine how the average and fluctuations of these currents can be derived from microscopic dynamics. In recent years, considerable progress has been made in this direction. First, it was found that current fluctuations have symmetries as expressed in the Gallavotti-Cohen [1,2] theorem. These symmetries are macroscopic manifestations of microscopic time reversibility. Second, Bodineau and Derrida formulated an additivity principle (AP) that allows one to calculate the whole distribution of current fluctuations once the first two cumulants are known [3]. The AP should hold for large, one-dimensional, diffusive systems and was validated in the symmetric exclusion process [4] and the Kipnis-Marchioro-Presutti (KMP) model of heat conduction [5]. More recently, it was also found to hold in three-dimensional deterministic models of heat conduction [6]. Independently, Bertini et al. developed a large deviation theory for density and current fluctuations in stochastic lattice gases $[7,8]$. The predictions of this hydrodynamic fluctuation theory coincide with those of the AP when the fluctuations are time independent. Interestingly, it was found that for sufficiently large fluctuations a dynamical phase transition can occur to a phase where density and current fluctuations become time dependent [8]. This transition was observed in a weakly asymmetric exclusion process on a ring [9] and more recently in the KMP model [10], also on a ring. This type of dynamical transitions can occur in situations that are not allowed in equilibrium and have been conjectured to be of relevance to such issues as breaking of chiral or CP symmetry [11].

In this paper, we study the current fluctuations in the weakly asymmetric exclusion process (WASEP) with open boundaries. We calculate the cumulant generating function of the current and the density profile giving rise to an atypical current using the AP. This extends earlier work [12]. We compare these results with those coming from calculations in finite systems using the density matrix renormalization group (DMRG). We recently showed how that approach, first introduced to study low temperature properties of quantum systems [13], can be applied to determine the cumulant generating function of the current or the activity of stochastic systems [14]. We find that for sufficiently large systems our results converge to those predicted by the $\mathrm{AP}$, further validating this principle. More interestingly, comparison of the two approaches allows us to formulate a finite size scaling theory for current fluctuations in the WASEP and compare it with known results for the symmetric exclusion process [15]. We also investigate the possibility of a dynamical phase transition but find no evidence for it.

\section{ADDITIVITY PRINCIPLE APPLIED TO THE WEAKLY ASYMMETRIC EXCLUSION PROCESS}

\section{A. Model}

In the asymmetric exclusion process (ASEP) [4], each site $i$ of a lattice of size $N$ can be empty or occupied by one particle. The dynamics is that of a Markov chain where particles jump to the right or left with different rates $(p$, respectively $q$ ). This describes the effect of an external field $E=\ln (p / q) / 2$. In this paper we will discuss the case where $p=1 / 2+v /(2 N), q=1 / 2-v /(2 N)$ referred to as the weakly asymmetric exclusion process (WASEP). This is a diffusive model where the AP should be applicable. We will consider the case of open boundaries where at its left (right) side the system is in contact with a reservoir at density $\rho_{a}\left(\rho_{b}\right)$. We will assume $\rho_{a}>\rho_{b}$ and $v>0$. The model will evolve to a nonequilibrium steady state (NESS).

\section{B. Current fluctuations from the additivity principle}

We are interested in the total number of particles or time integrated current $Q_{T}$ passing in a large time $T$ through the system. For $T$ sufficiently big, and using a continuum description in terms of $x=i / N \in[0,1]$, the average current equals

$$
\frac{\left\langle Q_{T}\right\rangle}{T}=-\frac{D(\rho)}{N} \frac{\partial \rho}{\partial x}+\frac{v \sigma(\rho)}{N} \equiv \frac{j^{*}}{N} .
$$

The first term is Fick's law and the second is the current due to the field $E$ in linear response. For the WASEP with the rates $p$ and $q$ as defined in the previous section, the diffusivity $D(\rho)=1 / 2$ and the mobility $\sigma(\rho)=\rho(1-\rho)$ [16] where $\rho(x)$ is the particle density.

In this paper, we are interested in the fluctuations of the current around the average value $j^{\star}$. For $T$ very large, the 
probability $P$ to observe an integrated current $Q_{T}=j T / N$ obeys a large deviation principle [17],

$$
P\left(\frac{Q_{T}}{T}=\frac{j}{N}, \rho_{a}, \rho_{b}\right) \sim \exp \left[\frac{T}{N} G\left(j, \rho_{a}, \rho_{b}\right)\right] .
$$

The large deviation function $G$ is zero at the average current $j^{*}$ of the NESS, and is strictly negative for other $j$ values. Hence for $T \rightarrow \infty$ only the average value can occur, but for finite $T$ fluctuations can occur with a probability determined by the large deviation function (LDF).

According to the AP [3], $G$ can be found from a variational principle,

$$
\begin{aligned}
G\left(j, \rho_{a}, \rho_{b}\right) & =-\min _{\rho(x)}\left[\int_{0}^{1} \frac{\left[j-v \sigma(\rho(x))+\frac{1}{2} d \rho / d x\right]^{2}}{2 \sigma(\rho(x))} d x\right] \\
& \equiv-\min _{\rho(x)} \int_{0}^{1} L\left[j, \rho_{a}, \rho_{b} ; \rho(x), \rho^{\prime}(x)\right],
\end{aligned}
$$

where the last line defines the Lagrangian $L$. The AP corresponds to the physical idea that locally the system is in equilibrium so that its fluctuations are Gaussian [9].

The profile minimizing the integral (3) can be obtained from an Euler-Lagrange equation,

$$
\frac{d}{d x} \frac{\partial L}{\partial \rho^{\prime}}=\frac{\partial L}{\partial \rho},
$$

where $\rho^{\prime}=d \rho / d x$. In this way, one finds that the optimal profile satisfies

$$
(2 \rho-1)\left[j^{2}-v^{2} \sigma^{2}(\rho)-\frac{1}{4}\left(\frac{d \rho}{d x}\right)^{2}\right]=\frac{1}{2} \sigma(\rho) \frac{d^{2} \rho}{d x^{2}} .
$$

Multiplying this equation by $(d \rho / d x) / \sigma^{2}(\rho)$ and realizing that $2 \rho-1=-d \sigma / d \rho$ one obtains

$$
-\frac{d \sigma}{d x}\left(\frac{j^{2}}{\sigma^{2}}-v^{2}\right)=\frac{1}{4} \frac{d}{d x}\left[\frac{1}{\sigma(\rho)}\left(\frac{d \rho}{d x}\right)^{2}\right] .
$$

The left hand side of this equation equals

$$
\frac{d}{d x}\left(\frac{j^{2}}{\sigma(\rho)}+v^{2} \sigma(\rho)\right) .
$$

Equation (5) can then be integrated. This gives

$$
j^{2}+v^{2} \sigma^{2}(\rho)=\left(\frac{1}{2} \frac{d \rho}{d x}\right)^{2}+\tilde{K} \sigma,
$$

where $\tilde{K}$ is an integration constant. Subtracting $2 j v \sigma(\rho)$ from both sides of this equation and defining $-2 K=\tilde{K}-2 j v$ one finally obtains

$$
\left(\frac{1}{2} \frac{d \rho}{d x}\right)^{2}=[j-v \sigma(\rho)]^{2}+2 K \sigma(\rho) .
$$

In order to solve this equation, we first assume that the profile is monotonically decreasing $(d \rho / d x<0)$ from $\rho_{a}$ to $\rho_{b}$. Taking the negative square root of (6) and integrating, one obtains

$$
\int_{\rho_{a}}^{\rho_{b}} \frac{d \rho}{\left\{[j-v \sigma(\rho)]^{2}+2 K \sigma(\rho)\right\}^{1 / 2}}=-2 .
$$

Solution of this equation for given $j$ and boundary conditions determines the integration constant $K$. Using the found value, one determines the density profile associated with this current from

$$
\int_{\rho_{a}}^{\rho} \frac{d \rho}{\left\{[j-v \sigma(\rho)]^{2}+2 K \sigma(\rho)\right\}^{1 / 2}}=-2 x .
$$

Second, inserting (6) in Eq. (3) gives after some elementary calculations the function $G$,

$$
\begin{aligned}
G\left(j, \rho_{a}, \rho_{b}\right)= & \int_{\rho_{a}}^{\rho_{b}}\left[\frac{[j-v \sigma(\rho)]^{2}+K \sigma(\rho)}{\left\{[j-v \sigma(\rho)]^{2}+2 K \sigma(\rho)\right\}^{1 / 2}}\right. \\
& -[j-v \sigma(\rho)]] \frac{d \rho}{2 \sigma(\rho)} .
\end{aligned}
$$

Instead of using the large deviation function (2), one can also describe the current fluctuations using the cumulant generating function (CGF),

$$
\mu\left(s, \rho_{a}, \rho_{b}\right)=\lim _{T \rightarrow \infty} \frac{1}{T} \ln \left\langle e^{s Q_{T}}\right\rangle,
$$

which is related to $G$ through a Legendre transform [17],

$$
\mu\left(s, \rho_{a}, \rho_{b}\right)=\frac{1}{N} \max _{j}\left[s j+G\left(j, \rho_{a}, \rho_{b}\right)\right] \equiv \frac{M\left(s, \rho_{a}, \rho_{b}\right)}{N} .
$$

The CGF is mathematically similar to a free energy, and by tuning $s$ one can select realizations of the Markov process with atypically large or small currents.

From (11), one finds that at the maximum

$$
s=-\frac{\partial G}{\partial j} .
$$

Using (9) this leads to

$$
s=\int_{\rho_{b}}^{\rho_{a}}\left[\frac{j-v \sigma(\rho)}{\left\{[j-v \sigma(\rho)]^{2}+2 K \sigma(\rho)\right\}^{1 / 2}}-1\right] \frac{d \rho}{2 \sigma(\rho)} .
$$

Inserting this result in Eq. (11) finally leads to an expression for the cumulant generating function,

$$
\begin{aligned}
M\left(s, \rho_{a}, \rho_{b}\right)= & \frac{1}{2} \int_{\rho_{b}}^{\rho_{a}} \frac{[j-v \sigma(\rho)] \nu-K}{\left\{[j-v \sigma(\rho)]^{2}+2 K \sigma(\rho)\right\}^{1 / 2}} d \rho \\
& +\frac{v}{2}\left(\rho_{b}-\rho_{a}\right) .
\end{aligned}
$$

To summarize, for given $v, \rho_{a}, \rho_{b}$, and $j$ we first determine $K$ from (7). This has to be done numerically. Then the density profile, the large deviation function, the parameter $s$, and the CGF can be obtained from numerical integration of respectively (8), (9), (13), and (14).

In certain regions of parameter space, solving (7) does not give a real solution. This indicates that the density profile has an extremum. In that case, the solution of the variational problem (3) can still be determined but leads to more complicated expressions which are discussed in the Appendix. Also the numerical solution of the resulting equations becomes more difficult but poses no fundamental problem. 


\section{DMRG APPROACH TO CURRENT FLUCTUATIONS}

We now want to determine the density profile and the CGF for finite systems to see whether and how they converge to those predicted by the AP. With standard simulation techniques it is difficult to generate atypical currents since they occur with exponentially small probability. A cloning technique that overcomes this problem was proposed in Refs. [18,19]. However, this technique becomes less accurate for large fluctuations [5]. Recently, we proposed a different approach to current fluctuations based on the DMRG, the ideas behind which we now briefly explain [20].

The probability $P(\mathcal{C}, t)$ to observe the exclusion process in a given microscopic configuration $\mathcal{C}$ evolves according to the master equation $\partial_{t} P(\mathcal{C}, t)=\sum_{\mathcal{C}^{\prime}} H\left(\mathcal{C}, \mathcal{C}^{\prime}\right) P\left(\mathcal{C}^{\prime}, t\right)$ where $H$ is the generator of the process. It is by now well established [4] that the CGF (10) can be obtained from a modified generator $H_{s}$, which is constructed from $H$ as follows. Let $\alpha\left(\mathcal{C}, \mathcal{C}^{\prime}\right)$ be $+1(-1)$ when in the transition from $\mathcal{C}^{\prime}$ to $\mathcal{C}$ a particle enters (leaves) the system on its left side. Otherwise $\alpha\left(\mathcal{C}, \mathcal{C}^{\prime}\right)=0$. The off-diagonal elements of $H_{s}$ are then given by $H_{s}\left(\mathcal{C}, \mathcal{C}^{\prime}\right)=$ $H\left(\mathcal{C}, \mathcal{C}^{\prime}\right) e^{s \alpha\left(\mathcal{C}, \mathcal{C}^{\prime}\right)}$ while the diagonal elements of $H$ and $H_{s}$ are equal. The CGF for a system of $N$ sites, $\mu\left(s, \rho_{a}, \rho_{b}, N\right)$, then equals the largest eigenvalue of $H_{s}$ [4].

Moreover, let $\left|R_{0}\right\rangle$ and $\left\langle L_{0}\right|$ be the associated right and left eigenvector. Consider a dynamical variable $b(\mathcal{C}(t))$ (like the density at a given site) which depends on the microscopic configuration in which the system is at time $t$. It can be shown that the current weighted time-average of $b$, defined as

$$
\langle b(\tau)\rangle_{s} \equiv \frac{1}{T} \frac{\left\langle\int_{0}^{T} b(\mathcal{C}(\tau)) e^{s Q_{\tau}} d \tau\right\rangle}{\left\langle e^{s Q_{T}}\right\rangle}
$$

for $T$ large equals $\left\langle L_{0}|\hat{b}| R_{0}\right\rangle$ where $\hat{b}$ is the operator associated to the variable $b$ [21]. On the other hand, the average of $b$ at the final time $T$, defined as

$$
\langle b(T)\rangle_{s} \equiv\left\langle b(\mathcal{C}(T)) e^{s Q_{T}}\right\rangle /\left\langle e^{s Q_{T}}\right\rangle,
$$

equals $\left\langle 0|\hat{b}| R_{0}\right\rangle$ where $\langle 0|$ is the projection state $\sum_{\mathcal{C}}\langle\mathcal{C}|$. So both the CGF and the density profiles can be determined from the largest eigenvalue of $H_{s}$, its eigenvectors, and the projection state. From a mathematical point of view, solving this problem is similar to that of determining the ground state and its eigenvector for a quantum spin chain, where the main difference is that in the stochastic problem the generator $H_{s}$ is not Hermitian. One of the most precise numerical approaches to determine ground state properties of quantum chains is the DMRG [13,22]. The first applications of this method to stochastic generators were presented in Refs. [23,24]. We recently showed that the DMRG also works well for generalized generators associated with current fluctuations [14]. Here we give also results on density profiles, both time-averaged and at the final time.

We can typically obtain reliable results up to $N \approx 120$. As a check of the DMRG approach we have calculated density profiles for the totally asymmetric exclusion process $(p=$ $1, q=0)$ at $s=0$ where exact results for finite $N$ exist [25] and have found perfect agreement. We are not aware of any exact results for the density of the ASEP in finite systems and for $s \neq 0$.

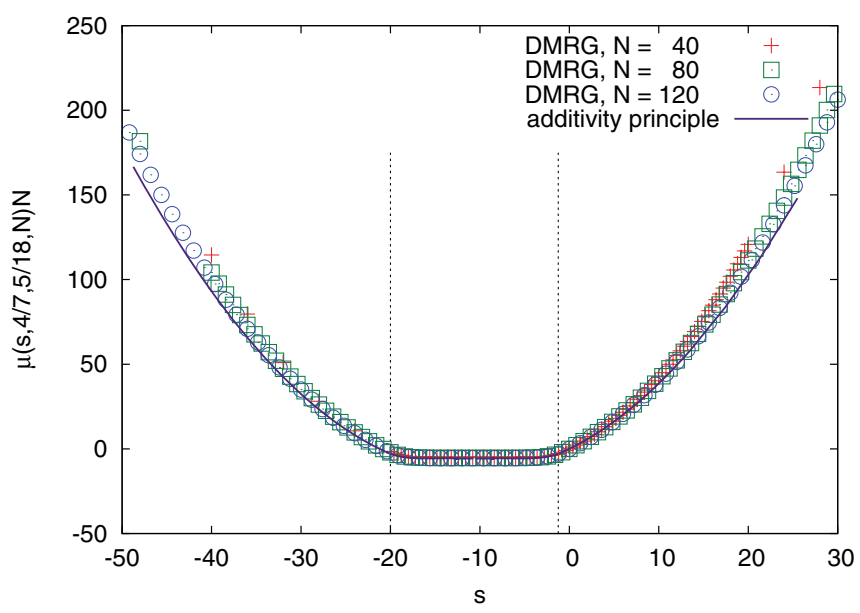

FIG. 1. (Color online) Cumulant generating function for the WASEP for $v=10, \rho_{a}=4 / 7, \rho_{b}=5 / 18$ from the additivity principle and from the DMRG.

\section{RESULTS}

We now compare the results obtained from the AP with those coming from the DMRG.

First, in Fig. 1 we present a typical result for the CGF. The full line is the prediction from the AP. In the regime between the vertical dotted lines, the optimal profile has a minimum, otherwise it is monotonically decreasing. The various symbols are DMRG results for $N \mu\left(s, \rho_{a}, \rho_{b}, N\right)$ at different $N$ values. As can be seen, for increasing system size, the AP and DMRG results coincide within numerical accuracy in an increasing range of $s$ values.

Within the whole $s$ region investigated our numerical data satisfy the Gallavotti-Cohen symmetry $\mu\left(s, \rho_{a}, \rho_{b}, N\right)=$ $\mu\left(\Delta-s, \rho_{a}, \rho_{b}, N\right)$ where [26]

$$
\Delta=-\ln \frac{\left(1-\rho_{a}\right) \rho_{b}}{\rho_{a}\left(1-\rho_{b}\right)}-(N-1) \ln \frac{1-v / N}{1+v / N} .
$$

A dynamical phase transition should show up as a point where the CGF becomes non analytical. On the scale of Fig. 1 this seems to occur where the profile changes from monotonic to one with a minimum. However, on closer examination, this turns out to be not the case. In Fig. 2 the second derivative of the CGF as calculated from the AP is plotted. This quantity shows only a finite peak and no discontinuities. Moreover the peaks are located close to but not exactly at the points where the form of the profile changes. Again the DMRG results (not shown) converge to those from the AP. This is for us a strong indication that there is no dynamical phase transition in the WASEP with open boundaries. We will come back to this issue in the next section.

In Fig. 3 we show the large deviation function $G(j)$ as calculated from the AP. For small deviations from the average current, we expect $P(j / N)$ to be Gaussian, so that the LDF is quadratic (crosses). Sufficiently large fluctuations are nonGaussian. Upon increasing $v$, we have found that the range of $j$ values where Gaussianity holds decreases.

We now turn to the density profiles. First, we observe that the time-averaged density profiles are invariant for the transformation $j \leftrightarrow-j$. This is a consequence of time 


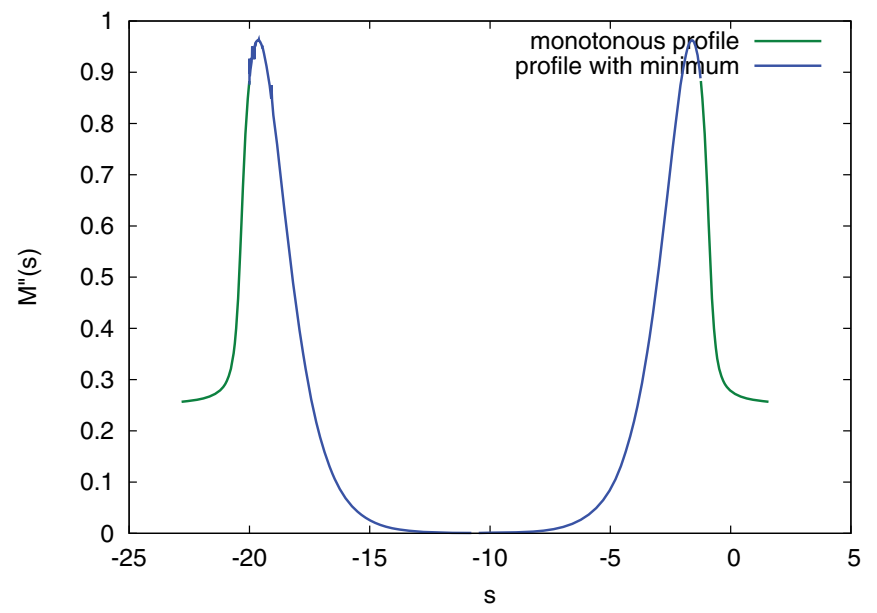

FIG. 2. (Color online) Second derivative of the cumulant generating function shown in Fig. 1. The results are from the AP.

reversibility, and is a special case of a more general result for isometric current fluctuations that holds in higher dimensions [27]. The invariance of the profiles follows directly from the equations of the AP, but is also valid for finite systems. Figure 4 shows a result for a system with $N=50$ and $j= \pm 1.5$. Density profiles calculated after a large time $T$ do not obey this symmetry.

For various values of $s$ (or $j$ ) we have calculated density profiles in finite systems. We show as an example in Fig. 5 the time-averaged profile corresponding to a large positive current fluctuation, $s=10$ (or $j=5.1214 \ldots$.). This is to be compared with the average current $(s=0)$ which at these parameter values equals $j^{*}=2.5845 \ldots$ The full line is the result from the AP; the symbols indicate DMRG data. As can be seen, the finite size results converge again to those predicted by the AP. This convergence is slowest near the boundaries. We therefore show in the inset an extrapolation of the average density at

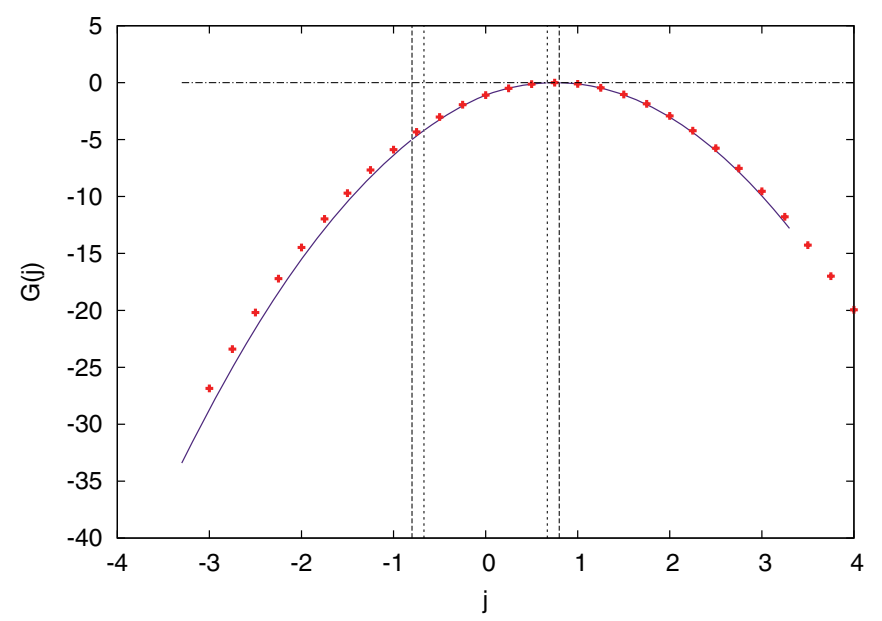

FIG. 3. (Color online) Current large deviation function for the WASEP for $v=3, \rho_{a}=4 / 9, \rho_{b}=5 / 13$ from the additivity principle (full line). The crosses give the results from a quadratic fit through the maximum. The vertical lines indicate regimes with a different density profile (left to right): profile with a maximum, monotonous profile, profile with a minimum, monotonous profile, profile with a maximum (see Fig. 7).

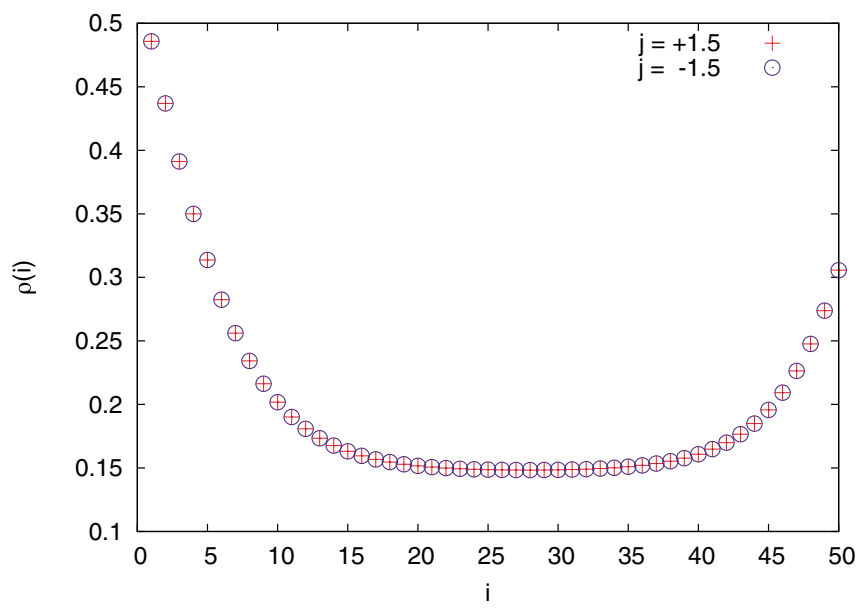

FIG. 4. (Color online) Time-averaged density profile in a finite system $N=50$ with current $j= \pm 1.5$ as calculated with the DMRG $\left(v=10, \rho_{a}=4 / 7, \rho_{b}=5 / 18\right)$.

the leftmost side $(i=1)$ for various $N$, which is consistent with the asymptotic prediction. The figure also shows that, apart from boundary effects, the density profile becomes flat and concentrated near $\rho=1 / 2$ in order to carry this current which is almost twice as large as the average one. In Fig. 6 we show similarly the density profile associated with a very small current, $s=-10, j=0.00041 \ldots$ Also for this profile with a minimum, finite $N$ results converge to the predictions of the AP. In this case the density becomes almost zero, except near the boundaries. In the inset we compare the time-averaged and the final time profile for a system with $N=120$. It is not known how to determine the final time profile from the AP [28]. Time-averaged and final time profiles are different in the same way as spatial boundaries give rise to differences between bulk and surface densities [21].

In Fig. 7, we finally show a density profile with a maximum. In this case, the current is slightly larger than the typical one at the given parameter values. At first sight, the convergence to

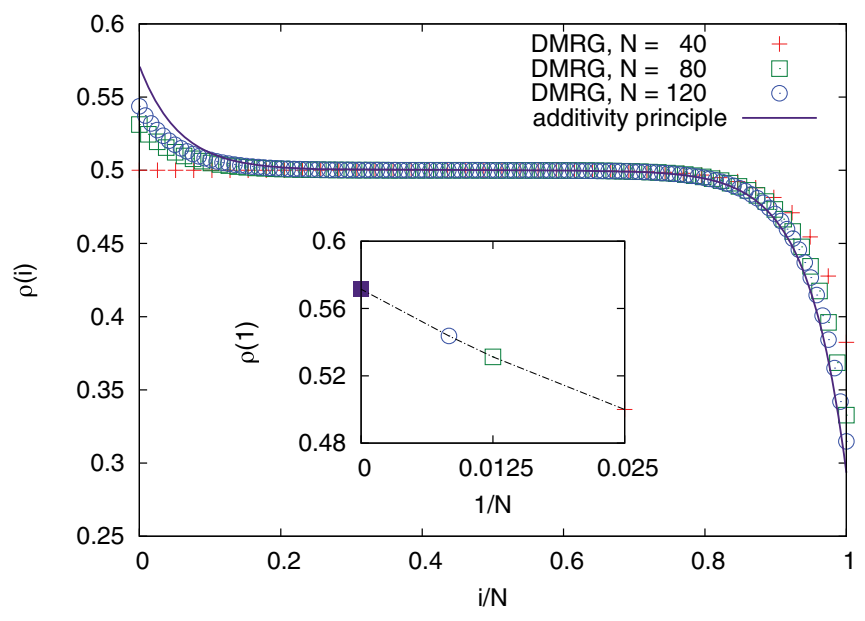

FIG. 5. (Color online) Time-averaged density profile at $s=10$ as calculated from the AP (full line) and from the DMRG. The inset shows the density at the first site as a function of $1 / N$ together with the value $\rho_{a}$ at the boundary (full square) $\left(v=10, \rho_{a}=4 / 7, \rho_{b}=\right.$ $5 / 18)$. 


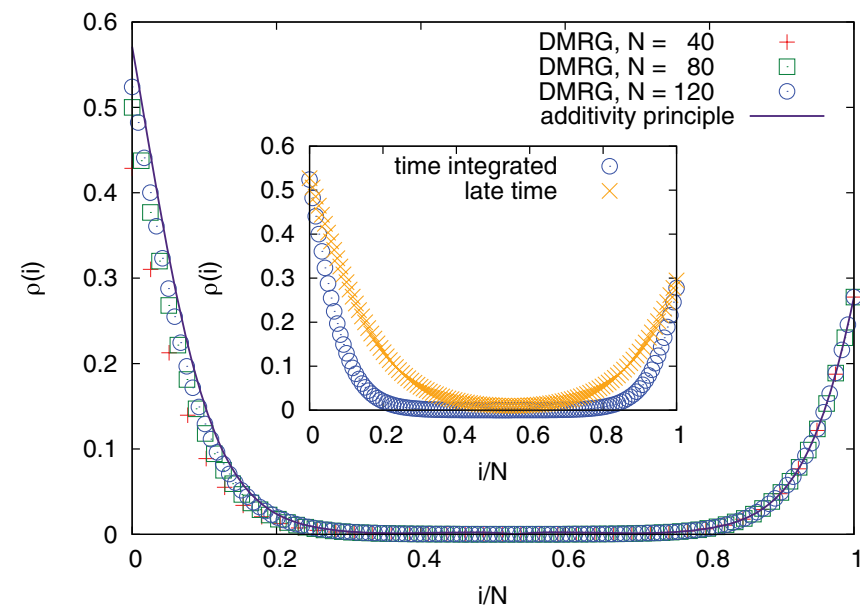

FIG. 6. (Color online) Time-averaged density profile at $s=-10$ as calculated from the AP (full line) and from the DMRG. The inset shows the time-averaged and the final time profile for $N=120(v=$ $\left.10, \rho_{a}=4 / 7, \rho_{b}=5 / 18\right)$.

the AP results seems slower, but notice that the vertical scale of this figure is different from that of the previous ones and that we could only reach $N=100$ with the DMRG.

\section{FINITE SIZE SCALING THEORY}

In the previous sections we have given results for the CGF of the WASEP, both for finite systems (DMRG) and in the hydrodynamic limit (AP). From a comparison of the two it is possible to get insight in the finite size scaling theory for the WASEP.

Such a theory was recently formulated for a class of diffusive systems [15] for which the diffusivity $D(\rho)$ is constant $(=D)$ and the mobility $\sigma(\rho)$ is quadratic in the density (so that the second derivative $\sigma^{\prime \prime}$ is also constant). For these systems it was shown that the CGF has the form (we drop the dependence of the CGF on the reservoir densities for simplicity

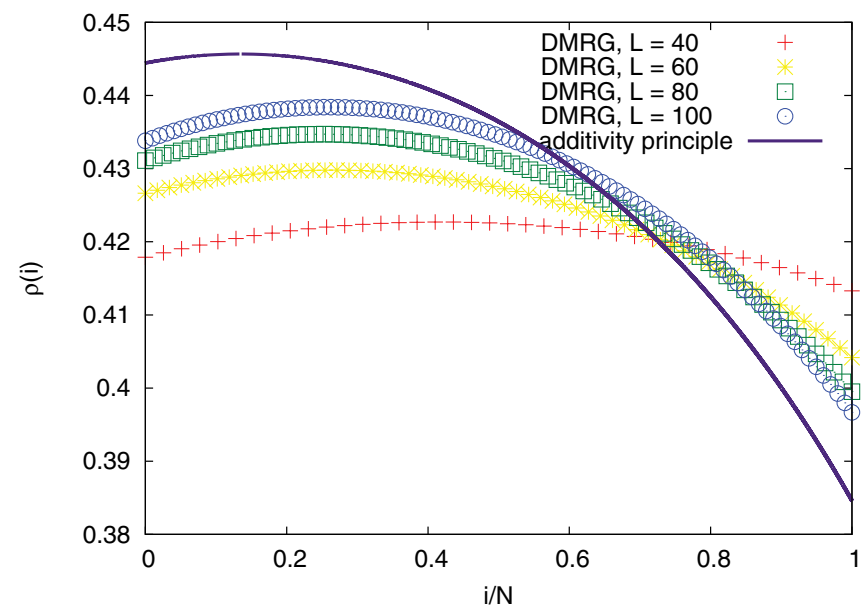

FIG. 7. (Color online) Time-averaged density profile for $j=$ $0.8376 \ldots$ as calculated from the AP (full line) and from the DMRG. $\left(v=3, \rho_{a}=4 / 9, \rho_{b}=5 / 13\right.$. Typical current for these parameter values: $\left.j^{\star}=0.7595 \ldots\right)$.

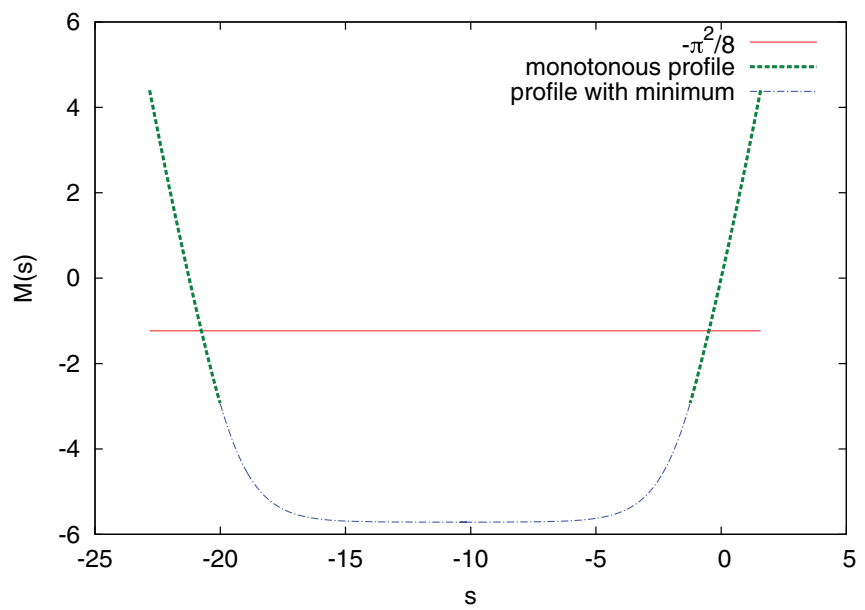

FIG. 8. (Color online) Cumulant generating function as calculated from the AP for $v=10, \rho_{a}=4 / 7, \rho_{b}=5 / 18$. The horizontal line lies at $M(s)=-\pi^{2} / 8$.

of notation)

$$
\begin{aligned}
\mu(s, N)= & \frac{1}{N} M_{d}(s)+\frac{D}{8 N^{2}} \mathcal{F}\left(\frac{\sigma^{\prime \prime}}{2 D^{2}} M_{d}(s)\right) \\
& +\frac{c}{N^{2}} M_{d}(s)+O\left(N^{-3}\right) .
\end{aligned}
$$

Here $M_{d}(s)$ is a function that has to be calculated separately for each (diffusive) model. It has been determined for the symmetric exclusion process (SEP) [29] and the KMP model [15]. It gives the leading term of the CGF for $N \rightarrow \infty$. The other two terms represent finite size corrections. Here, $c$ is a model-dependent constant. Most interestingly, the second term represent a universal finite size correction given in terms of the function

$$
\mathcal{F}(u)=-4 \sum_{q=n \pi, n \geqslant 1}\left(q \sqrt{q^{2}-2 u}-q^{2}+u\right) .
$$

This function has a singularity when its argument approaches $\pi^{2} / 2$. This singularity gives rise to a (dynamical) phase transition. For the symmetric exclusion process, $D=1 / 2$ and $\sigma=\rho(1-\rho)$ so that the singularity is crossed when $M_{d}(s)=-\pi^{2} / 8$. It can however be shown that this value is never reached. So, for the SEP with open boundaries, no dynamic phase transition occurs.

Since the WASEP is also a diffusive model we first investigate whether the same finite size scaling theory can be applied to it. Comparing the CGF as calculated using the AP, Eq. (11), with the general expression (18), allows us to identify $M_{d}(s)$ for this case with the function $M\left(s, \rho_{a}, \rho_{b}\right)$ calculated in Sec. II. Interestingly, in this case, for $v$ sufficiently large, there is an interval of $s$ values where $M(s)<-\pi^{2} / 8$. For example, in Fig. 8, we replot the function $M(s)$ as calculated using the AP (same parameter values as in Figs. 1 and 2). The horizontal line is located at $-\pi^{2} / 8$. Therefore, if the function $\mathcal{F}(u)$ would appear in the finite size scaling theory of the WASEP, a phase transition should occur. However, as we already discussed in the previous section we see no numerical evidence for this. This leads us to conclude that the finite size scaling corrections to the WASEP are not given in terms of the function $\mathcal{F}(u)$. 


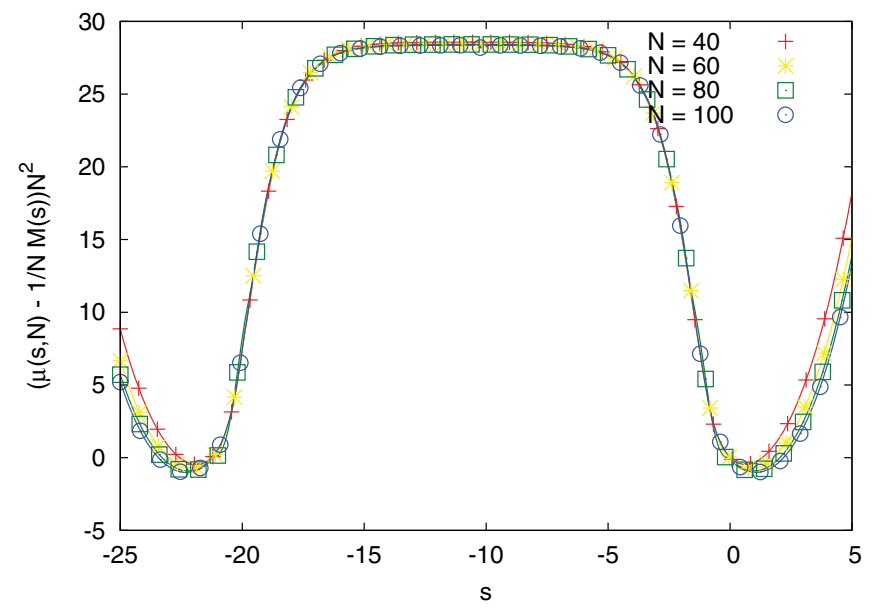

FIG. 9. (Color online) Test of the scaling ansatz (21) for the $\operatorname{WASEP}\left(v=10, \rho_{a}=4 / 7, \rho_{b}=5 / 18\right)$.

Given the mathematical similarity between the CGF and a free energy, it is natural to propose that both functions have similar scaling properties. Consider therefore an equilibrium system where $t=\left(T-T_{c}\right) / T_{c}$ and $h$ are temperature and field variables that measure the distance from a critical point. The singular part of the free energy per site, $f_{s}(t, h, N)$, then has a finite size scaling of the form

$$
f_{S}(t, h, N) \approx \frac{1}{N^{d}} \mathcal{K}\left(N^{y_{t}} t, N^{y_{h}} h\right),
$$

where $\mathcal{K}$ is a scaling function. Given the similarity between the CGF and a free energy we recently proposed [14] that the CGF should have a finite size scaling of similar form. In particular we conjecture that

$$
\mu(s, N)=\frac{1}{N} M_{d}(s)+\frac{1}{N^{z}} \mathcal{H}\left(N^{y_{s}-1} s\right) .
$$

The first term is a regular contribution while the second contains the finite size corrections and can be singular. The variable $s$ plays a role analogous to a temperature [see (10)] and we associate a scaling exponent $y_{s}$ with it [30]. $\mathcal{H}$ is a scaling function and $z$ is the dynamical exponent of the model. It replaces the dimension $d$ occurring in Eq. (20). This is because time replaces number of particles in the large deviation theory of nonequilibrium phenomena [17].

In particular, for diffusive models, the result (18) is consistent with this form with $y_{s}=1$ and $z=2$ (as should be for a diffusive model). We have also found agreement with the conjecture (21) for the case of the totally asymmetric exclusion process where $z=y_{s}=3 / 2[14,31]$.

We have therefore checked whether our results for the WASEP are consistent with the proposal (21) with $z=2, y_{s}=$ 1. This implies that the combination $[\mu(s, N)-M(s) / N] N^{2}$ is a function of $s$ only. In Fig. 9 we show a typical result. As can be seen, our data are clearly consistent with the expected scaling form and the graph for large $N$ gives the function $\mathcal{H}$ numerically. So far it has not been possible to calculate this function analytically, following, for example, the approach of [15].

\section{CONCLUSIONS}

In this paper we have calculated the current fluctuations of the WASEP and their associated density profiles using two approaches. The additivity principle gives results in the thermodynamic limit assuming that the density profile is time independent. The DMRG gives numerically exact results for finite systems with $N$ up to $\approx 120$. Comparing the two approaches, we have shown that current and density fluctuations in the WASEP converge for $N$ sufficiently large to those given by the AP. In contrast to the case on a ring [9] no evidence for a dynamic phase transition was found. In this respect, the WASEP behaves similarly to the SEP. Furthermore by using the results from both approaches we have gained insight in the finite size scaling theory of the current fluctuations and have found it to be of the form (21).

Recently, exact results for the cumulants of the current in the asymmetric exclusion process were determined [32]. In principle it should be possible, by taking appropriate limits, to get analytical insight also in the WASEP case. Such an approach could give the form of the finite size scaling function $\mathcal{H}$.

In the last decade it has become clear that large deviation principles are essential in formulating a fluctuation theory out of equilibrium. For many models, however, an exact solution is not possible. We believe that the DMRG method in combination with "hydrodynamic" approaches such as the additivity principle will be an important tool to quantify fluctuations, formulate finite size scaling theories, and investigate universality classes for systems far from equilibrium.

\section{ACKNOWLEDGMENTS}

We would like to thank V. Lecomte for useful discussions and correspondence. We also thank J. Liesenborgs for help with the numerical solution of the equations coming from the AP.

\section{APPENDIX}

Here we discuss the solution to the variational problem (3) for a profile with a minimum. The case of a maximum is solved in a completely analogous way.

For a minimum, the density decreases $(d \rho / d x<0)$ from $\rho_{a}$ at the left boundary to $\rho_{m}$ at some $x_{m}\left(0<x_{m}<1\right)$. At $x_{m}$, $d \rho / d x=0$. The density increases again from $\rho_{m}$ at $x_{m}$ to $\rho_{b}$ at the right boundary $(d \rho / d x>0)$. We thus get from (6)

$$
\begin{aligned}
& {\left[j-v \sigma\left(\rho\left(x_{m}\right)\right)\right]^{2}+2 K \sigma\left(\rho\left(x_{m}\right)\right)=0,} \\
& \int_{\rho_{a}}^{\rho_{m}} \frac{d \rho}{\left\{[j-v \sigma(\rho)]^{2}+2 K \sigma(\rho)\right\}^{1 / 2}}=-2 x_{m}, \\
& \int_{\rho_{b}}^{\rho_{m}} \frac{d \rho}{\left\{[j-v \sigma(\rho)]^{2}+2 K \sigma(\rho)\right\}^{1 / 2}}=2\left(x_{m}-1\right) .
\end{aligned}
$$

For given $v, \rho_{a}, \rho_{b}$, and $j$ this set of equations has to be solved to determine $x_{m}, \rho_{m}$, and $K$. This was done using a Monte Carlo based algorithm. The profile is then determined from 
integration of (6). For $x \in\left[0, x_{m}\right],(8)$ remains valid while for $x \in\left[x_{m}, 1\right]$, one has

$$
\int_{\rho_{b}}^{\rho} \frac{d \rho}{\left\{[j-v \sigma(\rho)]^{2}+2 K \sigma(\rho)\right\}^{1 / 2}}=2(x-1) .
$$

In order to determine the large deviation function $G$ one inserts the expression for $d \rho / d x$ coming from (6) into (3) taking the negative root in $\left[0, x_{m}\right]$ and the positive one for $\left[x_{m}, 1\right]$. After some straightforward calculations this leads to

$$
\begin{aligned}
G\left(j, \rho_{a}, \rho_{b}\right)= & \int_{\rho_{a}}^{\rho_{m}}\left[\frac{[j-v \sigma(\rho)]^{2}+K \sigma(\rho)}{\left\{[j-v \sigma(\rho)]^{2}+2 K \sigma(\rho)\right\}^{1 / 2}}\right] \frac{d \rho}{2 \sigma(\rho)} \\
& +\int_{\rho_{b}}^{\rho_{m}}\left[\frac{[j-v \sigma(\rho)]^{2}+K \sigma(\rho)}{\left\{[j-v \sigma(\rho)]^{2}+2 K \sigma(\rho)\right\}^{1 / 2}}\right] \frac{d \rho}{2 \sigma(\rho)} \\
& -\int_{\rho_{a}}^{\rho_{b}}[[j-v \sigma(\rho)]] \frac{d \rho}{2 \sigma(\rho)} .
\end{aligned}
$$

As in the main text, the parameter $s$ is still given by (12) so that from (A4) we find

$$
\begin{aligned}
s= & -\int_{\rho_{a}}^{\rho_{m}} \frac{j-v \sigma(\rho)}{\left\{[j-v \sigma(\rho)]^{2}+2 K \sigma(\rho)\right\}^{1 / 2}} \frac{d \rho}{2 \sigma(\rho)}+\int_{\rho_{a}}^{\rho_{b}} \frac{d \rho}{2 \sigma} \\
& -\int_{\rho_{b}}^{\rho_{m}} \frac{j-v \sigma(\rho)}{\left\{[j-v \sigma(\rho)]^{2}+2 K \sigma(\rho)\right\}^{1 / 2}} \frac{d \rho}{2 \sigma(\rho)} .
\end{aligned}
$$

Inserting this value in Eq. (11) finally gives

$$
\begin{aligned}
M\left(s, \rho_{a}, \rho_{b}\right)= & \frac{1}{2} \int_{\rho_{m}}^{\rho_{a}} \frac{[j-v \sigma(\rho)] v-K}{\left\{[j-v \sigma(\rho)]^{2}+2 K \sigma(\rho)\right\}^{1 / 2}} d \rho \\
& +\frac{1}{2} \int_{\rho_{m}}^{\rho_{b}} \frac{[j-v \sigma(\rho)] v-K}{\left\{[j-v \sigma(\rho)]^{2}+2 K \sigma(\rho)\right\}^{1 / 2}} d \rho \\
& +\frac{v}{2}\left(\rho_{b}-\rho_{a}\right) .
\end{aligned}
$$

After determination of $K, \rho_{m}$, and $x_{m}$ from (A1)-(A3), we can then obtain the large deviation function, the density profile, and the cumulant generating function from numerical integration of the appropriate equations.
[1] G. Gallavotti and E. G. D. Cohen, Phys. Rev. Lett. 74, 2694 (1995).

[2] J. L. Lebowitz and H. Spohn, J. Stat. Phys. 95, 333 (1999).

[3] T. Bodineau and B. Derrida, Phys. Rev. Lett. 92, 180601 (2004).

[4] B. Derrida, J. Stat. Mech.: Theor. Exp. (2007) P07023.

[5] P. I. Hurtado and P. L. Garrido, Phys. Rev. Lett. 102, 250601 (2009); Phys. Rev. E 81, 041102 (2010).

[6] K. Saito and A. Dhar, Phys. Rev. Lett. 107, 250601 (2011).

[7] L. Bertini, A. De Sole, D. Gabrielli, G. Jona-Lasinio, and C. Landim, Phys. Rev. Lett. 87, 040601 (2001).

[8] L. Bertini, A. De Sole, D. Gabrielli, G. Jona-Lasinio, and C. Landim, Phys. Rev. Lett. 94, 030601 (2005).

[9] T. Bodineau and B. Derrida, Phys. Rev. E 72, 066110 (2005).

[10] P. I. Hurtado and P. L. Garrido, Phys. Rev. Lett. 107, 180601 (2011).

[11] G. Jona-Lasinio, Prog. Theor. Phys. 124, 731 (2010).

[12] T. Bodineau and B. Derrida, J. Stat. Phys. 123, 277 (2006).

[13] S. R. White, Phys. Rev. Lett. 69, 2863 (1992).

[14] M. Gorissen, J. Hooyberghs, and C. Vanderzande, Phys. Rev. E 79, 020101(R) (2009); M. Gorissen and C. Vanderzande, J. Phys. A 44, 115005 (2011).

[15] A. Imparato, V. Lecomte, and F. van Wijland, Phys. Rev. E 80, 011131 (2009).

[16] H. Spohn, Large Scale Dynamics of Interacting Particles (Springer, Berlin, 1991).

[17] H. Touchette, Phys. Rep. 478, 1 (2009).
[18] C. Giardinà, J. Kurchan, and L. Peliti, Phys. Rev. Lett. 96, 120603 (2006).

[19] V. Lecomte and J. Tailleur, J. Stat. Mech.: Theory Exp. (2007) P03004.

[20] M. Gorissen, Ph.D. thesis, Hasselt University, Diepenbeek, 2011.

[21] J. P. Garrahan, R. L. Jack, V. Lecomte, E. Pitard, K. van Duijvendijk, and F. van Wijland, J. Phys. A 42, 075007 (2009).

[22] U. Schollwöck, Ann. Phys. 326, 96 (2011).

[23] Y. Hieida, J. Phys. Soc. Jpn. 67, 369 (1998).

[24] E. Carlon, M. Henkel, and U. Schollwöck, Eur. Phys. J. B 12, 99 (1999).

[25] B. Derrida, M. R. Evans, V. Hakim, and V. Pasquier, J. Phys. A 26, 1493 (1993).

[26] J. de Gier and F. H. L. Essler, J. Stat. Mech. (2006) P12011.

[27] P. I. Hurtado, C. Pérez-Espigares, J. J. del Pozo, and P. L. Garrido, Proc. Natl. Acad. Sci. USA 108, 7704 (2011).

[28] B. Derrida (private communication).

[29] B. Derrida, B. Douçot, and P.-E. Roche, J. Stat. Phys. 115, 717 (2004).

[30] In the present paper we counted the number of particles flowing through one bond of the lattice. In Ref. [14] the current was counted through all bonds. In that case the argument of the scaling function in Eq. (21) becomes $N^{y_{s}} s$.

[31] B. Derrida and J. L. Lebowitz, Phys. Rev. Lett. 80, 209 (1998); B. Derrida and C. Appert, J. Stat. Phys. 94, 1 (1999).

[32] M. Gorissen, A. Lazarescu, K. Mallick, and C. Vanderzande, Phys. Rev. Lett. 109, 170601 (2012). 